\section{FASTER, MORE EFFECTIVE WOUND HEALING}

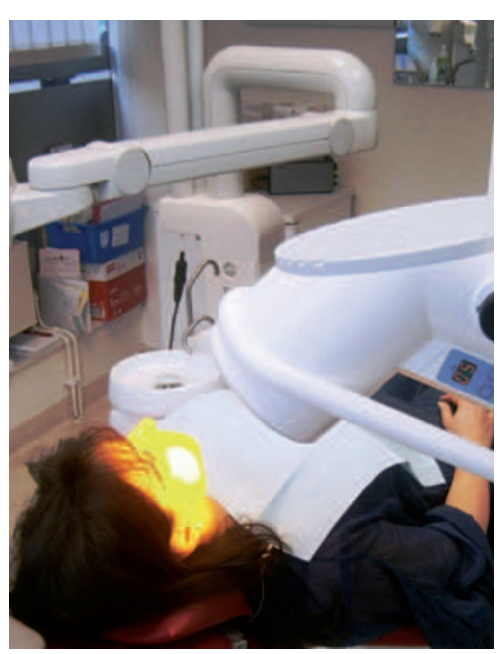

With the IPPL-SYSTEM (BLT)

treatment programme you can treat patients faster and more effectively.

IPPL stands for Incoherence -

Polarised - Polychromatic - Low energy light and is a patented light therapy and certified as a medical device (93/42/EEC). This optical device emits a light similar to the electromagnetic spectrum that is produced naturally by the sun without ultraviolet (UV) radiation. It functions at a cellular level and is regenerative, based on the four different properties and characteristics of the interaction.

IPPL has a wavelength between $480 \mathrm{~nm}$ and 3,400 $\mathrm{nm}$ and no UV radiation is emitted. This method has been shown to be effective in apical periodontitis, periodontitis, gingivitis and sialos, healing after extraction, for improved osseointegration and for the healing of dental implants, reducing the use of antibiotics. Treatment with IPPL light heals the wound 1.6 times faster and improves circulation in the gingiva and mucosa which is effective in periodontal treatment. IPPL light has immunological effects, is accepted in the medical treatment of wound healing and is effective in the clinic and at home.

Contact info@galomedical.co.uk for further information about the IPPL-SYSTEM treatment programme and how it can be a real practice builder and increase your dental practice's profits.

\section{OUTSTANDING DIGITAL IMAGING SOLUTIONS}

Clark Dental offers a range of outstanding digital imaging solutions designed to keep you at the forefront of dental technology. This includes Schick 33 - the latest addition to the Schick Digital Imaging System.

Featuring the industry's highest image resolution and lowest learning curve, Schick 33 includes powerful DICOM digital radiography software, to give you a level of diagnostic control unlike ever before.

Schick 33 has a special clinical-specific mapping feature so you can quickly and simply change between presets for general dentistry, endodontics, periodontics or restorative dentistry. You can also save personalised presets for yourself and for your colleagues, which makes the system ideal for multi-clinician practices.

To find out more contact Clark Dental today. If you already own Schick products, upgrading to include Schick 33 is easy. You can also trade in your existing system up to 50\% with Clark Dental's new trade-in programme designed to help you stay ahead of the game.

Telephone Clark Dental on 01268733146 or visit www. clarkdental.co.uk.

\title{
ADD LONG-LASTING COLOUR AND SURFACE GLOSS
}

Due to be launched in July 2014, GC's Optiglaze Color is light-cured characterisation coating for indirect composite and acrylic restorations.

Everything looks better in Color - whether you are a dental technician looking for a simple solution to characterise your composite creations or a dentist wanting to add depth to your chairside milled restorations, the versatility of OPTIGLAZE Color will certainly impress you.

GC OPTIGLAZE Color are ready to use light-curing nano-filled coating liquids that give you the opportunity to add long-lasting colour and surface gloss in an extremely easy way.
GC OPTIGLAZE Color is set apart by the following features:

- A wide variety of colours

- Adds natural gloss and brilliance

- Thin 25-50 $\mu \mathrm{m}$ coating layer

- Long-lasting gloss

- High discolouration resistance

- High wear resistance

- Excellent bonding durability.

For full

details of these courses, or for further information, contact GC UK on 01908218999.

\section{GUTTA-PERCHA WITH INNER STRENGTH}

Maintaining its position at the forefront of obturation technology, DENTSPLY has introduced GuttaCore, the first crosslinked gutta-percha obturator. This wellestablished scientific process of crosslinking effectively bonds a stabiliser to the molecular structure of gutta-percha to offer a unique inner strength.

Fast, efficient and heating in seconds, the hydraulic force of GuttaCore sends warm gutta-percha flowing equally in three dimensions right down to the apex in one single insertion. The continually tapered design matches the shapes created by modern files, allowing for accurate cleaning, shaping and irrigation to facilitate the flow of GuttaCore in the root canal system.
For complete simplicity, clinicians can remove the handle by bending to either side of the canal wall without affecting the seal, as well as create post space. Safe, strong and biocompatible, the crosslinked GuttaCore can be easily removed and leaves no plastic core behind, saving you time and hassle and simplifying retreatment.

If you're looking for the next 3D generation in warm obturation technology, look no further than GuttaCore. To find out more contact DENTSPLY UK on 0800072 3313 or visit www.dentsply.co.uk. 